\title{
Metabolic Syndrome: Gender Differences
}

\author{
Elisa Lodi, Alberto Carollo, Valentina Martinotti and Maria Grazia Modena* \\ University of Modena and Reggio Emilia, Italy
}

*Corresponding author: Maria Grazia Modena, University of Modena and Reggio Emilia, Italy, Tel: +39 059 4224241/44878;

Email: mariagrazia.modena@unimore.it

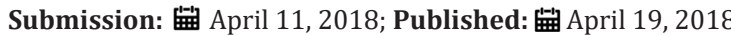

\section{Introduction}

Metabolic syndrome is commonly defined as the association of multiple risk factors for the development of atherosclerotic disease. Despite the different definitions of diagnostic criteria, the presence of at least three of the following conditions defines the clinical presentation of the metabolic syndrome: atherogenic dyslipidemia (low levels of HDL cholesterol, high levels of triglycerides, apolipoprotein B and small and dense LDL), basically high levels of systolic and diastolic blood pressure, impaired fasting glycemia and abdominal obesity [1]. Primary pathogenic mechanisms underlying metabolic syndrome are mainly due to insulin resistance (or poor insulin sensitivity) and visceral obesity. Insulin resistance, a condition where insulin determines a lower biological effect than expected, is largely represented in patients with metabolic syndrome and it is associated with a high risk of developing type 2 diabetes in the short term. Obesity and physical inactivity represent predisposing conditions for the development of metabolic syndrome and perhaps represent the main mechanism underlying the epidemic of metabolic syndrome, as observed in recent years.

Other precipitating factors of the development of the syndrome are represented by advanced age, hormonal imbalances, such as those observed during menopause. Patients diagnosed with metabolic syndrome have a doubled risk of atherosclerotic disease. Some authors questioned the utility of the diagnosis of metabolic syndrome in the management of prevention and definition of individual cardiovascular risk, focusing the attention on the individual assessment of cardiovascular risk factors. Actually, the coexistence of risk factors in the metabolic syndrome confers a much higher risk than the value simply obtained summing the individual factors. A further advantage deriving from the identification of a cluster of risk factors is observed in the syndrome, is represented by the greater attention paid to an early correction of lifestyle. Thus, the diagnosis of metabolic syndrome allows to the identification of subjects at risk of developing cardiovascular disease at an early stage. Furthermore, when adopting diagnostic criteria, it is necessary to take into account their simple applicability which involves the execution of few and targeted routine laboratory tests and simple anthropometric measurements. The prevalence of metabolic syndrome is exponentially increasing across the planet, as reflecting the parallel increase in obesity, expected to continue increasing in the next decade.

It is incredible to see that, while a part of the population suffers consequences of hunger, an increasingly part suffers consequences of obesity. One of the most discussed aspects in the various diagnostic classifications is the adjustment of the waist circumference based on the demographic characteristics of the examined population. In our country, data from population studies show a prevalence of the syndrome, defined by the ATP III criteria [1], ranging from 2 to $31 \%$ in adult men and from 1 to $35 \%$ for adult women.

Numerous observational and prospective studies have shown a different impact and distribution of cardiovascular disease in both sexes; in fact, women seem to develop cardiovascular events 10 years later than men with a marked increase of incidence in the age range mostly corresponding to the beginning of the menopausal period. But this gap is restricting, and it precisely may be due also to the metabolic syndrome. Among the cardiovascular risk factors to which women are exposed during their lifetime, age, sex and possible genetic substrate are not modifiable, while most of the risk factors such as arterial hypertension, dyslipidemia, obesity, glucose intolerance, cigarette smoking, diabetes mellitus and sedentariness are broadly reversible conditions. Importantly, among these, the first 4 represent components of the metabolic syndrome, a condition associated with a risk 5 times greater than developing diabetes and 3 times greater than developing cardiovascular death secondary to infarction or stroke [2]. In particular, abdominal obesity and the associated insulin resistance can contribute to the progression of dyslipidemia, to an increase in oxidative stress and therefore to endothelial dysfunction and vascular inflammation, leading to a prothrombotic state and accelerated atherosclerosis, in association with a large series of metabolic alterations.

We have at this point to clarify the difference between sex and gender: sex includes everything related to biology (genes, hormones, etc.), while the concept of gender, brought to the biomedical and human sciences, includes cultural, environmental and social influence [3]. Post-menopausal women very often 
develop metabolic syndrome, much more then men of same age: this is an example of gender difference mainly due to environment and life- style. According to the definitions of metabolic syndrome reported by ATP III and IDF respectively, the prevalence of metabolic syndrome in postmenopausal women in the EUROASPIRE study was $56 \%$ and $72 \%$ respectively, compared to $40 \%$ and $59 \%$ of men [4]. Such a high prevalence is supported by the fact that menopause is associated with an increase in sedentariness as well as a significant increase in the visceral obesity rate facilitated by estrogen deficiency, which contributes to the development of dyslipidemia, insulin resistance with reduced glucose tolerance, increased of pressure levels, sympathetic hyperactivity and proinflammatory and prothrombotic state [5].

It has been observed in parallel, how these metabolic alterations are more prevalent in post- menopause and in men over 50 years of age, and that the prevalence of the already elevated metabolic syndrome during the menopausal transition, undergoes a further increase in the postmenopausal period (SWAN study) [5]. Insulin resistance and visceral obesity seem to play a primary role in the pathogenesis of the metabolic syndrome in the post-menopausal phase, which, after a progressive reduction during the transition phase, is separate from an insulin sensitivity index markedly lower than that of the premenopausal period, regardless of the presence of obesity and plasma levels of blood sugar and fasting insulin (Figure 1). In confirmation of the central role of estrogenic deficiency in the genesis of this phenomenon, in humans no similar changes in insulin sensitivity have been observed in the same age group. A longitudinal study on 543 women in the perimenopausal period followed for 6 years, showed an absolute increase in fat mass of $3.4 \mathrm{~kg}$ and an increase in the waist circumference equal to $5.7 \mathrm{~cm} \mathrm{[6].} \mathrm{In} \mathrm{confirmation} \mathrm{of} \mathrm{the} \mathrm{relationship} \mathrm{between} \mathrm{menopause}$ and metabolic alterations, these parameters are proportionally correlated with the FSH plasma levels. Furthermore, the increase in visceral fat is inversely related to insulin sensitivity and associated with an increase in triglycerides, free fatty acids and small and dense LDL. Visceral obesity is the condition responsible for the pathophysiological relationship between insulin resistance and metabolic syndrome in females and is associated with an increased production of adipokines such as leptin, adiponectin and resist in.

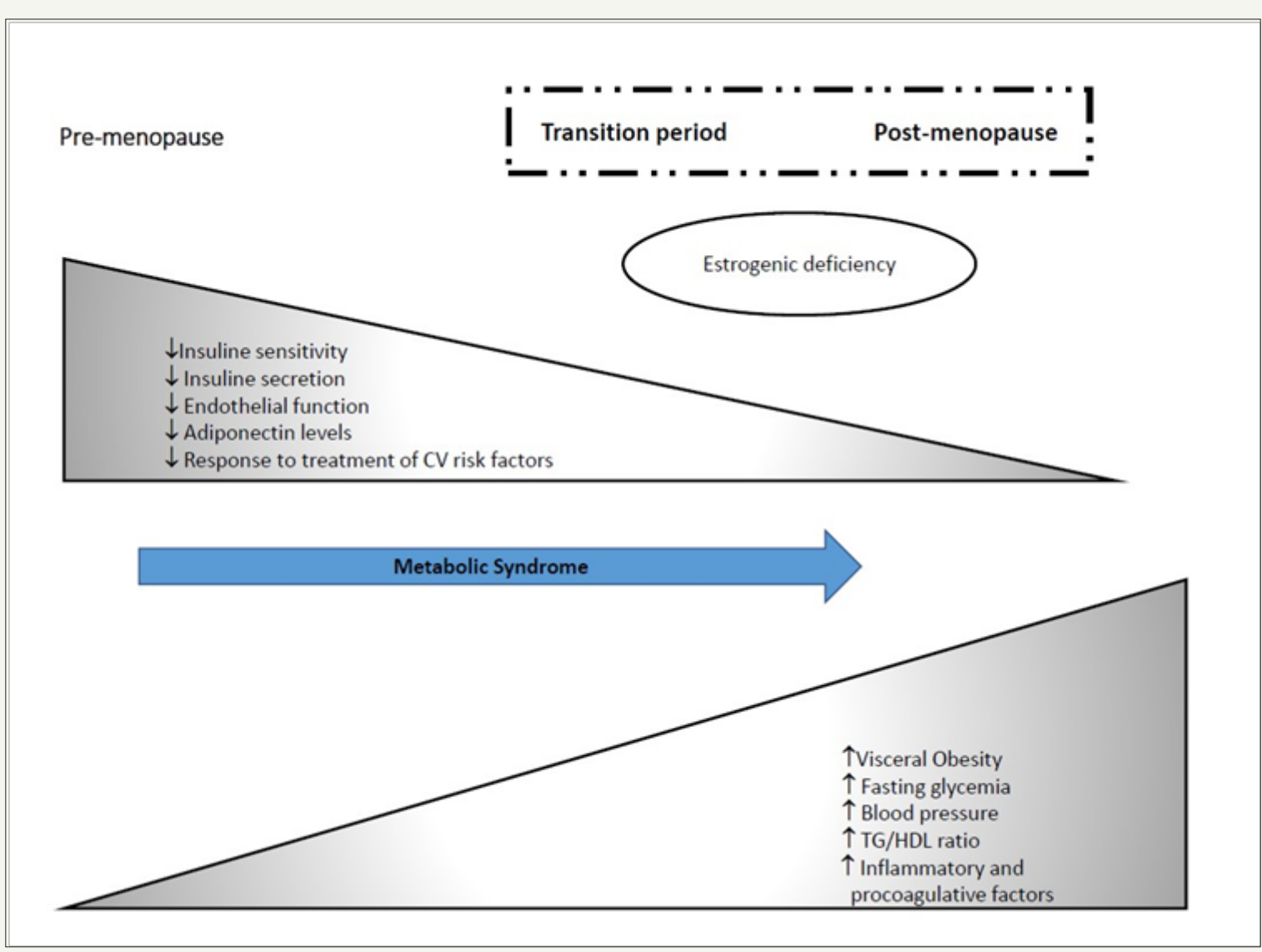

Figure 1:

These proteins, synthesized by adipocytes, bind to receptors mainly present in brain, endothelial and vascular walls. In the transition from premenopausal to postmenopausal period, leptin levels progressively increased, indicating that visceral fat can determine a pro- inflammatory and pro-coagulation state such as observed in patients with metabolic syndrome, through the secretion of this peptide. In contrast, plasma adiponectin levels were lower in the postmenopausal period and inversely correlated 
with insulin sensitivity [7]. The systemic inflammatory status observed in postmenopause is associated with a worsening of endothelial function assessed as flow-mediated dilation of the brachial artery. Endothelial dysfunction, present since the early stages of the menopausal period, is associated with a greater development of arterial hypertension after a follow-up of 3.6 years [8]. Furthermore, our study has shown that post-menopausal hypertension associated with metabolic syndrome, compared to a similar condition without metabolic syndrome, is associated with greater organ damage, worse endothelial function and is more resistant to therapy [9].

Given the central role of estrogenic deficiency in determining the metabolic changes observed in menopause, several studies have evaluated the impact of hormone replacement therapy on various components of the metabolic syndrome. However, while reducing estrogen, abdominal obesity, insulin resistance, the development of new cases of diabetes, the LDL/HDL cholesterol ratio, blood pressure values and inflammatory indices, they increased protein $\mathrm{C}$ and reduced protein $\mathrm{S}$, showing a potential prothrombotic effect [10]. Therefore, the total overall effect of postmenopausal hormone therapy seems to be an increase in arterial and venous thrombotic events (data from the WHI and HERS studies), so it is no longer recommended, and the suspension is rather preferable for some categories at risk. More than $50 \%$ of women over the age of 60 years present current diagnostic criteria of the metabolic syndrome with an increased cardiovascular risk that arouses strong social and economic concerns. A programmatic effort must focus on nutrition education, physical activity and implementation of the therapeutic strategies recommended by the guidelines to substain healthcare and social costs. The drugs should be able to control the risk factors, to improve and not to worsen the insulin resistance and, possibly, to act also on other conditions associated with the metabolic syndrome, such as small and dense LDL, microalbuminuria, oxidative stress, endothelial dysfunction and pro-coagulation status.

\section{References}

1. Miccoli R, Bianchi C, Odoguardi L, Penno G, Caricato F, et al. (2005) Prevalence of the metabolic syndrome among Italian adults according to ATP III definition. Nutr Metab Cardiovasc Dis 15(4): 250-254.

2. Holvoet P, Lee DH, Steffes M, Gross M, Jacobs DR (2008) Association between circulating oxidized low-density lipoprotein and incidence of the metabolic syndrome. JAMA 299(19): 2287-2293.

3. Principle of Gender-Specific Medicine-Gender in the Genomic Era.

4. Pyörälä K, Lehto S, De Bacquer D, De Sutter J, Sans S, et al. (2009) Risk factor management in diabetic and non- diabetic patients with coronary heart disease. Findings from the EUROASPIRE I AND II surveys. Diabetologia 47(7): 1257-1265

5. Janssen I, Powell LH, Crawford S, Lasley B, Sutton-Tyrrell K et al. (2008) Menopause and the metabolic syndrome: the study of women's health across the nation. Arch Intern Med 168(14): 1568-1575.

6. Sowers MF (2000) The menopause transition and the aging process: a population perspective. Aging 12(2): 85-92.

7. Murdock KW, LeRoy A, Fagundes C (2018) Inhibition is associated with metabolic syndrome and depression trough inflammation. Stress and Health 2018: 1-5.

8. Carey DG, Jenkins AB, Campbell LV, Freund J, Chisholm DJ (1996) Abdominal fat and insulin resistance in normal and overweight women: Direct measurements reveal a strong relationship in subjects at both low and high risk of NIDDM. Diabetes 45: 633-638.

9. Modena MG, Molinari R, Muia N, Castelli A, Pala F, et al. (1999) Double-blind randomized placebo-controlled study of transdermal estrogen replacement therapy on hypertensive postmenopausal women. Am J Hypertens 12(10): 1000-1008.

10. Modena MG, Origliani G, Nuzzo AC, Rossi R (2008) Metabolic syndrome affects cardiovascular risk profile and response to treatment in hypertensive postmenopausal women. Hypertension 52(5): 865-869.

11. Modena MG, Sismondi P, Mueck AO, Kuttenn F, Lignières Bd, et al. (2005) New evidence regarding hormone replacement therapies is urgently required transdermal postmenopausal hormone therapy differs from oral hormone therapy in risks and benefits. Maturitas 52(1): 1-10.
Creative Commons Attribution 4.0 International License

For possible submissions Click Here
Submit Article

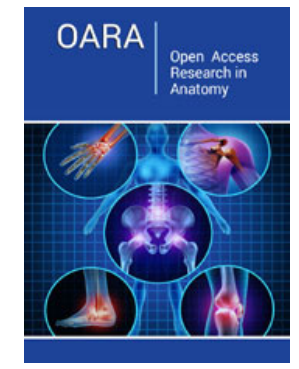

\section{Open Access Research in Anatomy}

\section{Benefits of Publishing with us}

- High-level peer review and editorial services

- Freely accessible online immediately upon publication

- Authors retain the copyright to their work

- Licensing it under a Creative Commons license

- Visibility through different online platforms 